\title{
Factores que influyen en la ingesta alimentaria de pacientes en diálisis peritonial del Hospital Guillermo Almenara Irigoyen, 2011.
}

\author{
Claudia SERRANO LUJÁN' y Neda POMÉ CÁRDENAS²
}

\begin{abstract}
RESUMEN
Objetivo: El objetivo de este estudio fue analizar la ingesta alimentaria de pacientes en diálisis peritonial y determinar la relación de la ingesta con diversos factores que pueden influenciar en ella. Metodología: Se evaluaron a 20 pacientes de DP del Hospital Guillermo Almenara, sin criterio de exclusión alguno. La evaluación nutricional fue completa, incluyendo síntomas importantes de la patología como solución de dializado, diuresis, antropometría Indice masa corporal (IMC), Pliege cutáneo tricipital (PCT), Circunferencia muscular del brazo (CMB), nivel de úrea, anamnesis nutricional, análisis de la ingesta alimentaria mediante cuestionario de frecuencia de consumo de alimentos, registro de ingesta alimentaria de 3 días y uso de la tabla de composición de alimentos del Perú (2009). Además, se consideró una evaluación sobre la disponibilidad económica y psicológica, todo ello para relacionarlo con la situación de la ingesta. Resultados: De los 20 pacientes, 12 fueron hombres y 8 mujeres, en su mayoría, de 35 a 60 años. Se registró una ingesta proteica de 30\%, lo que indica una ingesta deficiente de proteínas. Se encontró relaciones indirectas entre la uremia y dializado, con la ingesta alimentaria (r: -0.38 y -0.2 , respectivamente). Mediante prueba de Chi-cuadrado no se encontró dependencia entre nivel depresivo e ingesta alimentaria (sig. 0.77). Conclusiones: Este grupo de pacientes presentan deficiencias de proteínas, vitaminas y minerales en su aporte dietético. Para este grupo de pacientes, los factores que influyen sobre la ingesta fueron: uremia, el dializado, y disponibilidad económica.
\end{abstract}

Palabras clave: Ingesta alimentaria, porcentaje de adecuación, requerimientos nutricionales, nivel de depresión, dialisis peritoneal.

\begin{abstract}
Objective: The aim of this study was to assess dietary intake of PD patients and to determine the intake with a variety of factors that can influence it. Methodology: We evaluated 20 PD patients of Guillermo Almenara Hospital, without any exclusion criteria. Nutritional assessment was complete, including major symptoms of the disease as dialysate solution, diuresis, anthropometry (BMI, PCT, WBC), level of úrea, nutritional history, analysis of dietary intake by food frequency questionnaire, food, registration of dietary intake of 3 days and use of food composition table of Peru (2009). It was also considered a brief assessment of the economic and psychological availability, for all this relate to the situation of the intake. Results: Of the 20 patients, 12 were men and 8 women, mostly 35 to 60 years. Protein intake was recorded $30 \%$ indicating a poor dietary intake. Indirect relationships were found between uremia and dialysis, with dietary intake $(r=-0.38$ and -0.2 , respectively). By chi-square test found no dependence between depressive and intake level (sig. 0.77). Conclusion: These patients have deficiencies of protein, mainly vitamins and minerals in your dietary intake. For this group of patients, the factors that influence intake were: uremia, dialysis, and economic availability.
\end{abstract}

Keywords: Food intake, percentage of adequacy, nutritional requirements, level of depression, peritonial dialysis.

${ }^{1}$ Estudiante del $5^{\circ}$ año de Nutrición Humana, Universidad Peruana Unión.

${ }^{2}$ Magíster en Salud Pública, Docente de la Facultad de Ciencias de la Salud, Universidad Peruana Unión. 


\section{INTRODUCCIÓN}

La malnutrición proteico-energética en pacientes con insuficiencia renal crónica (IRC) es un problema importante de salud por la frecuencia y la elevada comorbilidad que conlleva (Daugirdas, et al. 2002). Su patogénesis es compleja y requiere un manejo terapéutico multidisciplinario.

La diálisis peritoneal (DP) es una alternativa de vida que mejora la calidad de vida de los pacientes con insuficiencia renal crónica (IRC). Las causas principales de muerte en estos pacientes son de orígenes cardiovasculares, infecciosas y problemas nutricionales.

La diálisis puede influir negativamente en el estado nutricional del paciente al añadirse factores directamente relacionados a la diálisis peritoneal (DP) como por ejemplo: la anorexia por elevaciones de uremia y/o la administración continua de glucosa mediante el dializado, la pérdida de proteínas y de aminoácidos que pasan al líquido peritoneal, aumento de la demanda metabólica, algunas restricciones alimentarias y/o alteración de factores psico-sociales.

La prevalencia de malnutrición en DP parece ser alta. Se ha estudiado en varios trabajos y se han mostrado cifras que van del 18 al 56\% de los pacientes. (Guerrero, 1990). Este amplio rango viene dado por los distintos métodos empleados para valorar y clasificar el estado de nutrición de un paciente, problema muy común y no resuelto, ya que existen varios métodos y muchos de ellos de difícil reproducción, por lo que la fiabilidad de los resultados no es completa. También influye el tiempo en DP, ya que la prevalencia de $\mathrm{MN}$ aumenta con éste y después del primer año (Ccastro, 2002). En tres recientes estudios, en los que emplearon el mismo método para valorar al paciente encontraron MN severa en 13-18\% de los pacientes estudiados. En un 25-30\% había signos de MN moderada y tan solo en 50-55\% mantenían un estado de nutrición adecuado. La desnutrición y los factores relacionados con dicha situación están fuertemente asociados con una mayor tasa de mortalidad en pacientes del programa de DP. (Sancho, 2001).

Dentro del origen multifactorial de la malnutrición en pacientes de DP, la ingesta alimentaria inadecuada es una causa importante. (Castro, 2002). Un gran porcentaje de pacientes en DP consumen una cantidad de energía y proteínas inferiores a lo recomendado.

El volumen del dializado en el peritoneo, también influye en la disminución de la ingesta alimentaria, así como la saciedad precoz que produce la absorción contínua de glucosa. Esto se evidencia con un estudio don- de se midió la ingesta de alimentos comparando la de los pacientes en DP con HD y controles. Se demostró que los pacientes en DP comían 206+70 y 190+76 gramos de alimentos con y sin diálisis, siendo la diferencia no significativa. En comparación, los de Hemodialisis diaria (HD) consumían 295+115 gramos y los controles $357+175$, siendo la diferencia significativa de estos últimos con los de DP. (Hylander, 2000).

Por otro lado, el aspecto psico-social es un factor muy relevante e influyente en el estado nutricional de los pacientes en DP, lo cual el especialista debe tener en cuenta al indicar el tratamiento. Existe un considerable porcentaje de problemas psicológicos en esta población, por la situación a la cual están sujetos. Un estudio analizó la calidad de vida de 60 pacientes en diálisis y trasplante, estableciéndose las asociaciones entre calidad de vida, depresión y ansiedad mediante la adaptación y validación del Cuestionario de Calidad de Vida y la medición de las dimensiones de funcionalidad física, psico-social y ocupacional. En el análisis descriptivo se halló que los trasplantados tienen mejores índices de calidad de vida a comparación de los de diálisis, siendo éstos, los más afectados. Se concluyó que la percepción de la calidad de vida debe ser considerada en la adecuación de la indicación médica. (Jiménez, 2002).

Hylander (2000), indica que la depresión es el problema psicológico más frecuente en los pacientes que realizan diálisis. En su estudio determinó que al menos un $25 \%$ de los pacientes en hemodiálisis y un 30\% de aquellos en diálisis peritoneal están deprimidos.

Debido a la correlación existente entre el estado nutricional y tasas de morbi-mortalidad de los pacientes en DP, es esencial una evaluación nutricional minuciosa al inicio del tratamiento y rutinariamente, considerando los diversos factores que influyen o, en su conjunto, determinan el estado nutricional. El estado nutricional está estrechamente relacionado a la ingesta calórica-proteica de la dieta. Por tanto, es importante evaluar la ingesta dietética de estos pacientes, para que se pueda mejorar el plan de dieta, considerando los factores que influyen en su composición y cantidad.

Una vez conociendo los factores que influyen en la ingesta alimentaria y desnutrición de pacientes en DP, debemos tomar las medidas preventivas necesarias para el tratamiento nutricional de estos pacientes.

Es importante que desde el primer momento, el paciente inicie una dinámica de asesoramiento dietético, valoración nutricional e intervención nutricional según necesidades, sin llegar a situaciones muy avanzadas en las que cualquier intervención pierde eficacia. El asesoramiento dietético tiene como objetivo informar al paciente de la 
importancia de la dieta en el conjunto del tratamiento, los beneficios y complicaciones a corto-medio y largo plazo de una dieta adecuada o no adecuada, cómo combinar los distintos alimentos para intentar no superar los límites recomendados, etc.

Es fundamental que el paciente no se sienta frustrado por restricciones estrictas y limitaciones-prohibiciones en una gran cantidad de alimentos, que condicione un abanico de opciones de alimentos poco atractiva y que favorezca una escasa ingesta global de nutrientes.

La presente investigación tuvo como objetivo evaluar la ingesta alimentaria de los pacientes en DP e identificar los principales factores que influyen en ella. De esta manera, el profesional especialista en nutrición podrá contar con información analizada de manera clara y contundente, respecto a qué consideraciones se debe tener al evaluar la ingesta alimentaria de un paciente en DP.

\section{MATERIAL Y MÉTODOS}

Estudio de diseño no experimental, tipo descriptivo, correlacional y de corte transversal. El estudio se realizó en el Servicio de Nefrología del Hospital Guillermo Almenara Irigoyen, durante los meses de Mayo-Junio del año 2011. Los datos se obtuvieron tras la evaluación a 25 pacientes de DP; para lo cual no se consideraron criterios de exclusión, pues la evaluación se daría a todos sin excepción.

Se realizó la evaluación nutricional de dos maneras: subjetiva y objetiva. En la evaluación subjetiva se le entrevistó al paciente respecto a datos personales de importancia, patología y síntomas, además de la recau- dación de información respecto al aspecto psico-social, específicamente el estado depresivo, y la disponibilidad económica que ellos tienen para adquirir sus alimentos.

La evaluación dietética se realizó mediante dos instrumentos ya conocidos, el Cuestionario de Recordatorio de 24 horas, y el de Frecuencia de consumo de alimentos. Para el recordatorio, se tomaron en cuenta los últimos 3 días consecutivos de ingesta, para luego extraer un promedio en cuanto a la ingesta.

Por otro lado, la evaluación objetiva consistió en la determinación de medidas antropométricas, tales como: Índice de Masa Corporal (IMC), mediante el peso y la talla, proporción de masa grasa y muscular a través de la medición de Pliegue Cutáneo Tricipital (PCT), Circunferencia Braquial (CB) y Circunferencia Muscular del Brazo (CMB). Además, la recaudación de los parámetros bioquímicos de mayor relevancia: albúmina, creatinina, úrea y proteínas totales, mediante la base de datos del sistema informático del servicio. Además de la solución de dializado que están recibiendo, para posterior cálculo de requerimiento calórico dietético.

Dentro del análisis estadístico, se realizó la determinación de estadísticos descriptivos más relevantes. Esto incluye la frecuencia y/o porcentaje de la muestra según situación nutricional, porcentaje de adecuación de la dieta, hábitos alimentarios, nivel depresivo, disponibilidad económica, y así, de las variables más importantes. Posteriormente, se llevaron a cabo los análisis de correlación y de Chi-cuadrado para las no paramétricas, con la finalidad para identificación de factores principales de la ingesta alimentaria, con un nivel de significancia del $95 \%$.

\section{RESULTADOS}

\section{Figura 1}

Distribución porcentual según diagnóstico médico.

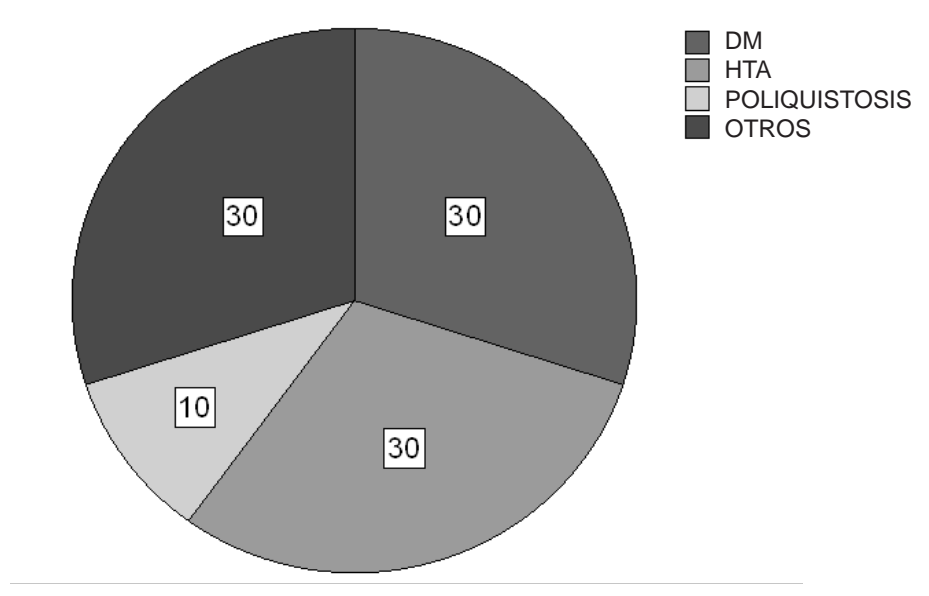

Se aprecia en la figura1, que los pacientes presentaron diversos diagnósticos médicos, como Diabetes Mellitus (DM) Poliquistosis, Hipertensión Arterial (HTA) y otros. Sin embargo, la mayoría de los pacientes presentaban DM y/o HTA. 
Figura 2

Diagnóstico nutricional según IMC

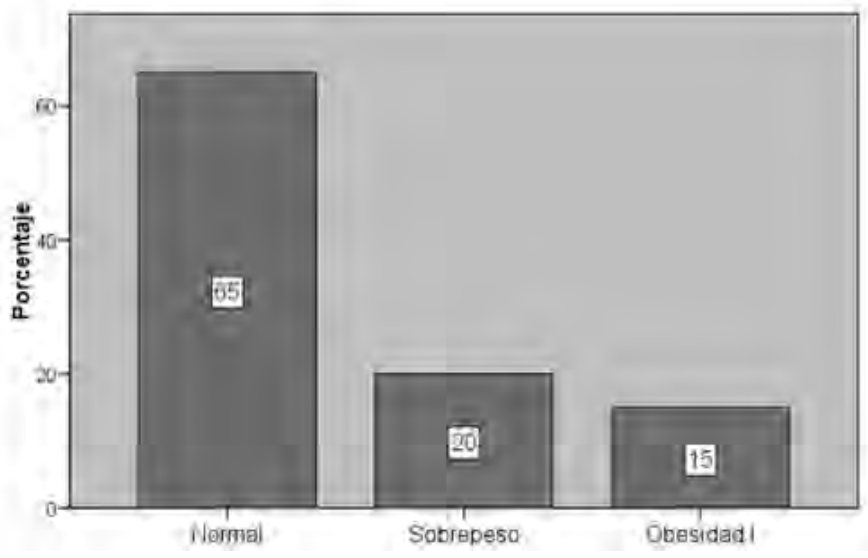

En la figura 2 se aprecia la evaluación nutricional mediante medidas antropométricas que se realizó a los pacientes según IMC, observándose a la mayoría con un diagnóstico normal (60\%), pero también una considerable proporción de sobrepeso y obesidad, con 20 y $15 \%$ respectivamente.

\section{Tabla 1}

\section{Porcentaje de adecuación de ingesta proteica}

\begin{tabular}{lcc}
\hline \multicolumn{1}{c}{ \% Adecuación } & Frecuencia & Porcentaje \\
\hline Bajo $(<90 \%)$ & 19 & 95.0 \\
Adecuado $(90-110 \%)$ & 1 & 5.0 \\
Total & 20 & 100.0 \\
\hline
\end{tabular}

Como se observa en la tabla 1, en el grupo de estudio se observó que solo un paciente cubría sus necesidades proteicas, y el resto (19 pacientes) presentaban un porcentaje de adecuación bajo. Mientras que la media de la ingesta proteica a través de la dieta fue de $0.66 \mathrm{~g} / \mathrm{kg}$ peso/día.

\section{Figura 3}

Nivel de estado depresivo

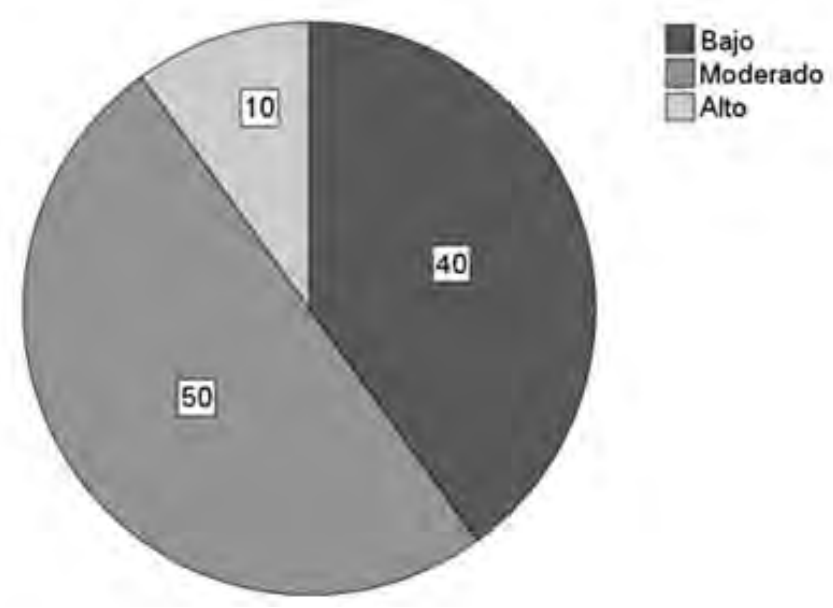

Según la figura 3, se aprecia que la mitad de los pacientes (10 personas) tenían un nivel moderado de estado depresivo, y un $10 \%$ presentaban un alto nivel de depresión. 


\section{DISCUSIÓN}

Tras la evaluación nutricional mediante medidas antropométricas que se realizó, se clasificaron a los pacientes según IMC (figura 2), observándose a la mayoría con un diagnóstico normal $(60 \%)$, pero también una considerable proporción de sobrepeso y obesidad, con 20 y $15 \%$ respectivamente.

Sin embargo, mediante otros parámetros antropométricos, como el PCT y el CMB, existe una prevalencia considerable de desnutrición. Así, según el PCT, tenemos al $60 \%$ en desnutrición, el $15 \%$ en estado normal y el $25 \%$ en exceso de reserva de grasa. Por otro lado, el diagnóstico según $\mathrm{CMB}$, indica relativamente lo mismo, el 35\% presenta desnutrición respecto a la reserva proteica.

De igual modo, el aporte proteico, muy relevante para el mantenimiento de las funciones vitales del organismo, no cubre las cantidades requeridas según fisiopatología de la DP, como muchos autores lo señalan (Montenegro, 2009), el consumo de proteínas deberá ser 1,1-1,3 g/kg peso/día, debido al alto grado de catabolismo y pérdida de proteínas que acontecen en el mecanismo de la DP, como se señaló anteriormente.

En el grupo de estudio, se observó que solo un paciente cubría sus necesidades proteicas, y el resto (19 pacientes) presentaban un porcentaje de adecuación bajo (tabla 1). Mientras que la media de la ingesta proteica a través de la dieta fue de $0.66 \mathrm{~g} / \mathrm{kg}$ peso/día.

Tras la evaluación de parámetros bioquímicos, se encontró que el 50\% del total mantenían niveles normales (100-150 mg/dl), mientras que el 10\% tenían niveles elevados y el resto (40\%), niveles inferiores. La uremia promedio de los pacientes fue de $107.9 \mathrm{mg} / \mathrm{dl}$, dando a entender que no existen cifras alarmantes de niveles anormales de uremia.

Gonzáles y Arango (2009), sostienen que uno de los factores que inducen a la baja ingesta de alimentos es la disminución de apetito debido a niveles elevados de úrea.

Frente a esta afirmación, se realizó un análisis de correlación entre ingesta calórica y el nivel de uremia, encontrándose un coeficiente de determinación (R) igual a $-0,377$, lo que nos indica una relación indirecta entre ambas variables, es decir, mientras más alto sea el nivel de úrea en sangre, menos será la ingesta alimentaria. Esta prueba fue significativa (sig. 0,050).
Otro de los factores que influyen en la ingesta alimentaria de pacientes con DP es el aspecto emocional. En los pacientes con insuficiencia renal terminal la depresión ha sido asociada con una mayor mortalidad en estos pacientes y el riesgo de hospitalización aumenta. (Lopes, 2002).

En nuestro estudio, se encontró que la mitad de los pacientes (10 personas) tienen un nivel moderado de estado depresivo, y un 10\% representan a un alto nivel de depresión (figura 3). Estas cifras, juntas, representan la mayoría de la muestra, como en riesgo de depresión o con diagnóstico de depresión.

Respecto a la glucosa absorbida mediante dializado, la mayoría de pacientes (55\%) reciben una cantidad de glucosa de $100-120 \mathrm{mg} / \mathrm{dl}$, siendo un $5 \%$ que recibe de $150 \mathrm{mg} / \mathrm{dl}$ a más y la media de $114.52 \mathrm{mg} / \mathrm{dl}$. En realidad, reciben mayores cantidades de glucosa en el dializado, pero de la glucosa recibida solo se absorbe aproximadamente el $70 \%$ del total de glucosa contenida en el dializado. Se realizó el cálculo a partir del contenido total de glucosa (Riella, 2006) .

Además, se realizó un análisis de correlación de Pearson, al 95\% de confiabilidad, entre las variables tratadas. Se encontró un valor de $\mathrm{R}=-0.282$ lo que nos indica que hay correlación indirecta lineal, es decir, cuanto más sea la cantidad de glucosa absorbida, menor será la ingesta alimentaria.

Sancho (2001), nos refiere que la principal causa por la que existe distensión abdominal acompañado de malestar y sensación de plenitud gástrica, es el volumen contínuo de líquido infundido en la cavidad peritoneal, y la absorción de glucosa, lo cual puede dificultar la ingesta alimentaria. Se evidencia la limitación de la ingesta oral, al tener de forma contínua niveles de glucosa e insulina superiores a los habituales en los períodos de ayuno, por lo que no se estimula el apetito.

\section{CONCLUSIONES}

El 30\% de pacientes evaluados presentaron una ingesta calórica inadecuada (menor al 90-110\% del RDA). La ingesta proteica promedio de los pacientes fue de $0.66 \mathrm{~g} / \mathrm{kg} /$ día, lo que significa un bajo aporte proteico (normal: $1-1,3 \mathrm{~g} / \mathrm{kg} / \mathrm{día})$. La mayor proporción $(75 \%)$ de pacientes consumen carnes, frecuentemente y en las cantidades requeridas.

Los principales factores influyentes en la ingesta alimentaria de los pacientes fueron: niveles de uremia, dosis de dializado y la disponibilidad económica para la adquisición de alimentos. 


\section{REFERENCIAS BIBLIOGRÁFICAS}

1. Aguilera, A., Marrón, B., \& Ortiz, A. Patogénesis de la malnutrición en pacientes en diálisis con énfasis en los aspectos más específicos de la diálisis peritoneal. Dial Traspl. 2009; 30(2): 5762.

2. Castro M. J. (2002). Nutrición en pacientes con Diálisis Peritoneal. Estudio Transversal". Hospital La Paz, Madrid.

3. Daugirdas, John; Blake, Peter; Ing, Todd. (2003). Manual de diálisis. Editorial Masson. España, 431,432

4. Dombros E. V. "Is Malnutrition a problema for the patient on peritoneal dialysis? Perit Dial Int 15; Suplement 5; S10 - S19. 1995.

5. González M. T., (abril, 2009). Arango J. J. Concordancia entre índice de masa corporal y albúmina como método diagnóstico de desnutrición en pacientes en diálisis. Acta Med Colomb vol.31 No 2 .

6. Guerrero A. (1990). El estado de nutrición en el paciente urémico y en diálisis. Tesis doctoral. Universidad de Sevilla. 1990.

7. Gutiérrez C., Mayoral A., Velasco S.(2009). Prevalencia y detección de la desnutrición en pacientes en diálisis en la unidad de nefrología del Hospital General de Segovia. Rev. Nefrología. Vol. 12. № 4
8. Hylander B., Barkeling B., Rossner S. (2000). Eating behavior in continuous peritoneal dialysis and hemodialysis patients. Am Soc Kidney Dis; 20: 592-597.

9. Jiménez, S. (2002). Valoración del estado nutricional de pacientes en diálisis peritoneal con hipoalbuminemia. Unidad de diálisis peritoneal del Servicio de Nefrología. Complejo Hospitalario de Jaén.

10. Lopes A.(2002). Depression as a predictor of mortality and hospitalization among hemodialysis patients in United States and Europe". Kidney International. 62(1):199-207

11. Montenegro, J. (2009). Tratado de Diálisis Peritoneal. Editorial Elsevier. España.

12. Pecoits-Filho R, Lindholm B, Stenvinkel P. (2002). Malnutrición, inflamación y ateroesclerosis (MIA). Nephrol Dial Transplant.;17 Suppl 11:28-3.3.

13. Sancho, J.(2001). Factores de riesgos asociados con la supervivencia en pacientes en programa de diálisis peritoneal. España: Rev. Nefrología. Vol. XXI. Número 2.

14. Riella, M. (2006). Nutrición y riñon. Argentina: Editorial Médica Panamericana. 